\title{
OXYGEN ABUNDANCES IN LI-DEPLETED F-TYPE STARS
}

\author{
RAMON J. GARCLA LOPEZ, RAFAEL REBOLO, ARTEMIO \\ HERRERO AND JOHN E. BECKMAN \\ Instituto de Astrofísica de Canarias, E-38200, La Laguna, Tenerife, Spain
}

ABSTRACT We have obtained the oxygen abundance in a sample of 44 F-type dwarfs in the Ursa Major group and the Hyades open cluster, using an NLTE analysis of the O I infrared triplet at $\lambda \lambda 7771-7775 \AA$. These clusters show the "lithium gap" in the F spectral range, and it has been suggested that if this phenomenon were due to microscopic diffusion, their Li-depleted stars could also show oxygen and/or nitrogen depletion. Our results here show a substantial measure of uniformity in the oxygen abundances. The data might indicate a very small dip $(<0.1 \mathrm{dex})$ in the $\mathrm{O}$ abundance for stars located in the $\mathrm{Li}$ gap. Microscopic diffusion seems to be the only known mechanism able to produce an oxygen dip. If this mechanism were responsible for the $\mathrm{Li}$ gap, it would have to account for a depletion of up to two orders of magnitude in $\mathrm{Li}$ and, at the same time, less than 0.1 dex in oxygen. We note that turbulent mixing could modify a microscopic diffusion pattern in order to yield the observed abundances of the two elements.

\section{INTRODUCTION}

The strong dip in the lithium abundance found in F-type stars with ages greater than $\sim 10^{8}$ yrs, belonging to different open clusters and the field, in the effective temperature range 6400 to $6900 \mathrm{~K}$, is a phenomenon of considerable vaiue for studying the structure and evolution of $F$ stars. It was first discovered in the Hyades (Boesgaard and Tripicco 1986) and it is clearly present in the Ursa Major group (Boesgaard, Budge and Burck 1988). To explain the gap, several transport mechanisms have been proposed. These mechanisms can be divided into two different groups: those which invoke the nuclear burning of the $\mathrm{Li}$ atoms and those in which this is not necessary (see Garcia López and Spruit 1991). Microscopic diffusion (Michaud 1986) is contained in this latter group. Michaud (1988) suggested that if the observed $\mathrm{Li}$ depletion in the $\mathrm{F}$ stars were due to microscopic diffusion, we could expect to find a similar phenomenon for oxygen and/or nitrogen, which would not be predicted for the other mechanisms proposed. With this test in mind, we set about measuring the oxygen abundance in the $\mathrm{F}$ stars of the Hyades (age $\sim 6-8 \times 10^{8} \mathrm{yrs}$ ) and of Ursa Major (age $\sim 3 \times 10^{8}$ yrs.). 


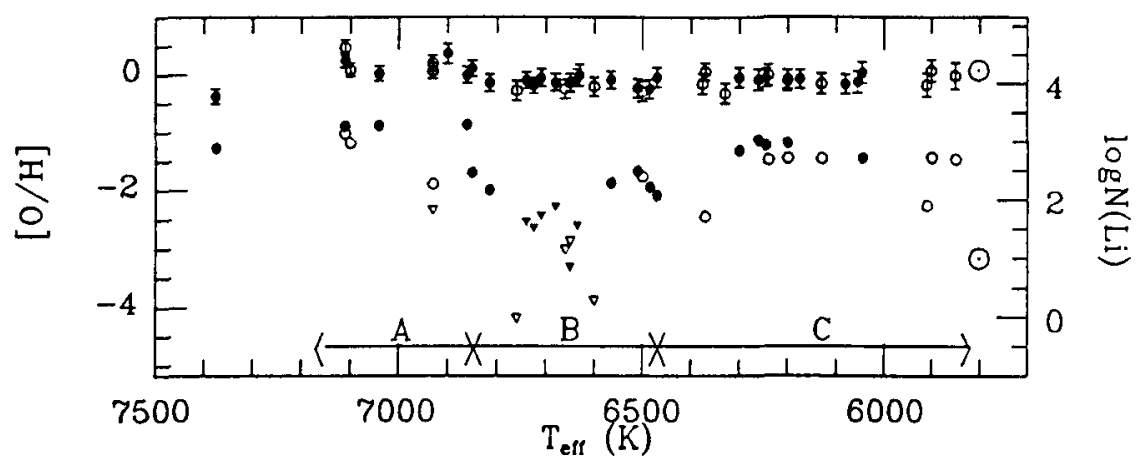

FIGURE I Oxygen and lithium abundances for the stars in our sample v. effective temperature. Filled circles: Hyades; open circles: Ursa Major group.

\section{DERIVATION OF THE ABUNDANCES}

The observations were performed during a series of observing runs on the 2.5 m Isaac Newton Telescope of the Observatorio del Roque de los Muchachos (La Palma) and the $2.2 \mathrm{~m}$ telescope of the Observatorio de Calar Alto (Almería). We selected the permitted triplet of oxygen I comprising the three lines at $\lambda \lambda$ $7771.944,7774.167$ and $7775.388 \AA$. The oxygen triplet was analyzed in NLTE, as the sensitivity of these lines to non-LTE effects is well known. For details of the analysis see García López et al. (1992).

\section{RESULTS AND DISCUSSION}

The results of our NLTE analysis of the infrared triplet are presented in Figure $I$, where we plot $[\mathrm{O} / \mathrm{H}]$ against $T_{\text {eff }}$. We also show the lithium abundances for the same stars. A notable feature of Fig. $I$ is that, in contrast to lithium, oxygen shows no major abundance "gap" in the effective temperature range considered. To examine the dependence of $[0 / H]$ on $T_{\text {eff }}$ we can divide the Figure into three different zones (neglecting the hottest star in the sample), viz: zone $A$, with $T_{\text {eff }}$ between 7150 and $6830 \mathrm{~K}$, zone $B$ between 6830 and $6450 \mathrm{~K}$, and zone $C$ between 6450 and $5800 \mathrm{~K}$. The mean abundances for the three zones are:

$$
\begin{array}{ccc}
\text { A } & \text { B } & \text { C } \\
+0.18 \pm 0.16 & -0.15 \pm 0.09 & -0.08 \pm 0.10 \\
9 \text { stars } & 17 \text { stars } & 17 \text { stars }
\end{array}
$$

where we have combined the data from the Hyades and the Ursa Major group. The quoted errors are the standard deviations with respect to the mean. We see that the mean abundance in group B is some $2 \sigma$ lower than that of group $A$, and $\sim 1 \sigma$ lower than for group C.

We must also draw attention here to the notable absence of oxygen in the hottest star of the sample VB38 $\left(T_{\text {eff }}=7375 \mathrm{~K}\right)$. This is related to the object being a chemically peculiar star, of type Am-Fm. VB38 figures as a well known 
Am star in the General Catalog of Ap and Am Stars recently published by Renson, Gerbaldi and Catalano (1991).

For the stars in temperature range B, the data show a slightly lower abundance, $0.07 \mathrm{dex}$, than the stars in the cooler range $C$. If there were a real underabundance of oxygen in stars of zone B compared with both cooler and hotter objects, this phenomenon would very probably be due to microscopic diffusion of the oxygen atoms below the base of the convection zone, which is the only known mechanism able to produce this kind of depletion in the abundance of a chemical element which is not destroyed by nuclear burning in the stellar interior. Our present oxygen measurements place a clear observational restriction on the working of the microscopic diffusion hypothesis to explain the $\mathrm{Li}$ gap: it must be capable of reducing the $\mathrm{Li}$ abundance by up to 2 orders of magnitude, while leaving the oxygen unaffected, or almost unaffected, with reductions less than 0.1 dex. These restrictions apply quantitatively, only, of course, to the two clusters observed which have ages less than $10^{9}$ years. Detailed computations for oxygen diffusion to compare directly with the observations are still required.

We note than the presence of turbulence will affect the simplest schemes of microscopic diffusion in which hydrostatic equilibrium is upset by an imbalance between gravitational and radiative accelerations. We can qualitatively suggest a possible scenario to explain the $\mathrm{Li}$ and $\mathrm{O}$ observations, in which this interaction would play a role. Given a degree of turbulence within the star, associated with rotation, or with rotational braking, or with the presence of internal gravity waves, the effect of the combination of microscopic diffusion and turbulence would be, in the $\mathrm{T}_{\text {eff }}$ range of the $\mathrm{Li}$ gap, to pull the $\mathrm{Li}$ atoms down to the level where they are destroyed by nuclear burning. On the other hand, the turbulent mixing would attenuate the effect of the microscopic diffusion on the $O$ atoms. Since these are not destroyed, mixing with the interior will not notably affect the surface abundance. If the slight dip in the $O$ abundance we appear to detect in range $B$ were real, this could be due to the residual effect of the microscopic diffusion, as would the slightly raised abundance in range $\mathrm{A}$. With observational effects of such small amplitude, however, more quantitative and testable conclusions must await more observations.

\section{REFERENCES}

Boesgaard, A. M., and Tripicco, M. J. 1986, Ap. J., 302, L49.

Boesgaard, A. M., Budge, K. G., and Burck, E. E. 1988, Ap. J., 325, 749.

García López, R. J., and Spruit, H. C. 1991, Ap. J., 377, 268.

García López, R. J., Rebolo, R., Herrero, A., and Beckman, J. E. 1992, Ap. J., submitted.

Michaud, G. 1986, Ap. J., 302, 650.

Michaud, G. 1988, in "Atmospheric Diagnostic of Stellar Evolution: Chemical Peculiarities, Mass Loss, and Explosion", IAU Coll. No. 108, Ed. K. Nomoto (Springer-Verlag), p. 3.

Renson, P, Gerbaldi, M., and Catalano, F. A. 1991, Astr. Ap. Supp., 89, 429. 